\title{
Correlative Chemical Element Imaging in Cells Using Fluorescence Microscopy and Synchrotron X-ray Fluorescence
}

\author{
Richard Ortega ${ }^{1}$, Stéphane Roudeau $^{1}$, Laura Perrin ${ }^{1}$ and Asuncion Carmona ${ }^{1}$ \\ 1. CENBG, CNRS, University of Bordeaux, 33175 Gradignan, France
}

Synchrotron radiation X-ray fluorescence (SXRF) microscopy holds the potential for fundamental breakthroughs in the understanding of biological systems because chemical element distributions can be evidenced at the sub-cellular level $(<100 \mathrm{~nm})$ with low detection limits $(<100 \mathrm{ng} / \mathrm{g})$. In addition to $\mathrm{SXRF}$, it is also possible to perform X-ray Absorption Spectroscopy (XAS) which is based on the photoelectric effect. X-ray absorption near edge structure (XANES) provides information about oxidation state and molecular geometry of the elements. What is the distribution of trace metals in cells? Do some elements accumulate within sub-cellular organelles? What are the chemical species of the elements in these organelles? These are some of the fundamental questions that can be addressed using X-ray chemical element imaging and speciation with synchrotron radiation beams [1].

The combination of SXRF with other imaging techniques enables comparing trace element distributions with subcellular structures, i.e. organelles, or protein distributions. Correlative microscopy is the best solution to provide reliable information about such subcellular localization of the elements. Several methodological approaches are possible. For example, SXRF has been combined to immunofluorescence microscopy [2] or to transmission electron microscopy [3]. Live cell fluorescence imaging prior to SXRF is also an interesting combination since it needs minimal sample preparation and therefore native trace element distributions can be preserved [4] (Fig. 1).

Examples of application can be divided according to the type of labeling used for live cell fluorescence microscopy. Some applications did not use any labeling but the auto-fluorescence properties of the organelles, such as in the case of the cytoplasm and chloroplast of aquatic protist cells [5], or the natural fluorescence of iodinated-polymyxin antibiotic drugs [6]. Subcellular fluorescent imaging can be performed easily with organelle specific organic fluorescent dyes. A wide variety of fluorescent stains are commercially available for organelles. Mitochondrial, lysosomal and nuclear dyes have been used to identify precisely these organelles prior to SXRF imaging [7-10]. Fluorescent-tagging of proteins, i.g. with GFP (green fluorescent protein), have also been applied in combination with SXRF for organelle identification such as for the endoplasmic reticulum [10] or the Golgi apparatus [11] (Fig. 2). In this late example, SXRF imaging was complemented with X-ray absorption spectroscopy for element speciation of oxidation state. GFP-tagged proteins are also valuable tools to locate metallo-protein inclusions such as for example in the study of amytropohic lateral sclerosis with cells overexpressing the $\mathrm{Cu}, \mathrm{Zn}$ superoxide dismutase [12]. Several studies have also reported the use of fluorescent metal sensors in the context of SXRF imaging at the single cell level, such as copper-selective metal sensors [13, 14] and zinc fluorophores [15].

This communication will review the applications of SXRF imaging combined to live cell fluorescence microscopy for element correlation with cellular structures or protein distributions. Protocols for optimal sample preparation and perspectives of instrumental developments for integrated correlative microscopy will be discussed. 
[1] MJ Pushie et al, Chem Rev 114 (2014) p. 8499-8541.

[2] R McRae et al, J Struct Biology 155 (2006) p. 22-29.

[3] Z Qin et al, J Synchrotron Radiation 19 (2012) p. 1043-1049.

[4] S Roudeau et al, Anal Bioanal Chem 406 (2014) p. 6979-6991.

[5] BS Twining et al, Anal Chem 75 (2003) p. 3806-3816.

[6] M Azad et al, Anal Chem 87 (2015) p. 1590-1595.

[7] T Bacquart et al, Anal Chem 79 (2007) p. 7353-7359.

[8] T Bacquart et al, Environ Res 110 (2010) p. 413-416.

[9] R McRae et al, Metallomics 5 (2013) p. 52-61.

[10] A Grubman et al, Chem Sci. 5 (2014) p. 2503-2516.

[11] A Carmona et al, Metallomics 6 (2014) p. 822-832.

[12] MW Bourassa et al, Frontiers in Aging Neuroscience 6 (2014) 110.

[13] L Yang et al, Proc Natl Acad Sci U S A. 102 (2005) p. 11179-11184.

[14] SC Dodani et al, Proc Natl Acad Sci U S A 108 (2011) p. 5980-5985.

[15] N McCormick et al, PLoS One 5 (2010) e11078.

[16] The authors acknowledge ESRF for beamtime allocation. The authors would also like to thank M.

Salomé, J. Susini, G. Veronesi, S. Bohic, R. Tucoulou, and P. Cloetens from ESRF.
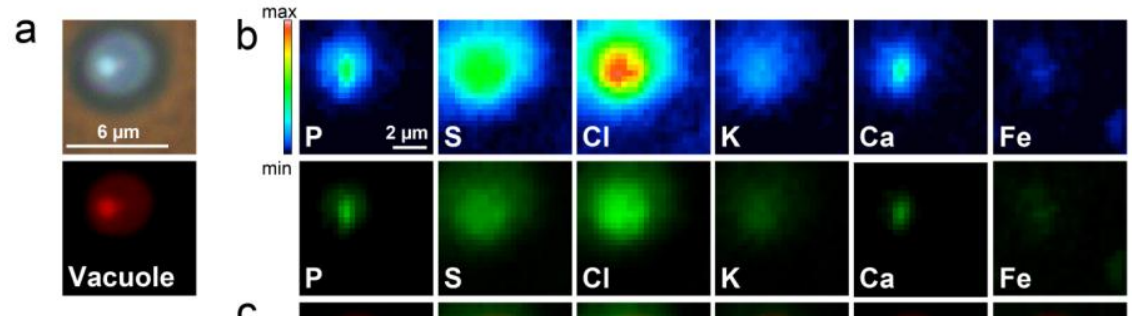

C

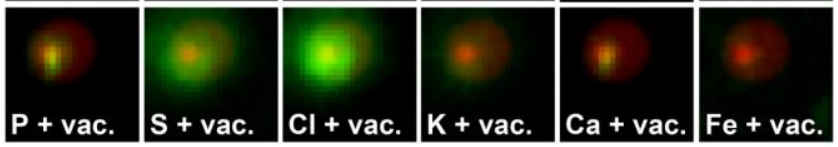

Figure 1. Correlative organelle fluorescence and SXRF microscopy of a yeast cell. a) yeast with ArgCMAC labeled vacuoles (blue fluorescence), and in false color (red) for the purpose of correlative imaging; b) SXRF chemical element distribution (ESRF ID21) (multi color bar) and same distribution maps in green for red green correlation; c) correlative imaging of SXRF (green) and vacuole fluorescence (red) showing the accumulation of $\mathrm{P}, \mathrm{Cl}$, and $\mathrm{Ca}$ within the yeast vacuole. Reproduced from [4], with permission from Springer.
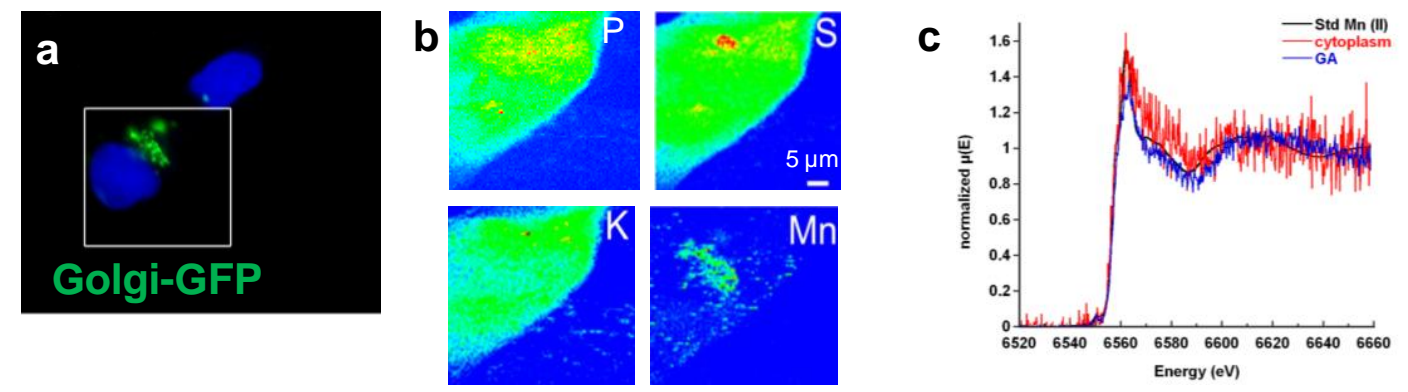

Figure 2. SXRF and XANES with organelle fluorescence identification a) live cell fluorescence imaging of Golgi apparatus (GFP, green) and nucleus (Hoechst dye, blue) in neuroblastic cells. b) SXRF element imaging (ESRF ID21 beamline) of the same cell after cryofixation (analysis performed at liquid nitrogen temp. in frozen hydrated state). c) Micro-XANES at Mn absorption K-edge in the cytoplasm and Golgi apparatus (GA) showing the presence of $\mathrm{Mn}(\mathrm{II})$ in both compartments. Adapted from [11]. 